\title{
Correlation of Cultural Intelligence of Managers and Their Effectiveness in a Copper Complex in South of Iran
}

\author{
Kobra Kahnouji \\ Social Determinants for Health Promotion Research center, \\ Hormozgan University of Medical Sciences, Bandar Abbas, Iran \\ Email: Kahnooji56@gmail.com \\ Rostam Pourrashidi \\ Associate Professor, Islamic Azad University of Kerman,Kerman. Iran \\ Email:Pourrashidi_28@yahoo.com \\ Shamsiyeh Ahangari \\ Department of Management, university of Sistan and Baluchestan, Zahedan. Iran \\ Email: ljbds@eco.usb.ac.ir

\begin{abstract}
*Ataollah Kahnouji
Kahnouji Ataollah, MSc, Islamic Azad University of Rafsanjan, Rafsanjan, Iran.

Email: atakahnoogi@yahoo.com
\end{abstract} \\ Sanjar Salajegheh \\ Associate Professor, Islamic Azad University of Kerman,Kerman. Iran \\ Email:Salajeghe_187@yahoo.com \\ Abdollah Kahnouji \\ PhD student, Islamic Azad University of Kerman,Kerman. Iran \\ Email:Kahnooji117@gmail.com
}

Doi:10.5901/mjss.2015.v6n4s2p708

\section{Abstract}

Cultural intelligence is one of the most efficient tools in doing tasks effectively in environments that have various and incompatible employees. Therefore, the purpose of the present study was to evaluate the relation of cultural intelligence of managers with their effectiveness in Sarcheshmeh Copper Complex. The study was conducted on 184 managers of Sarcheshmeh Copper Complex and the questionnaires were distributed among them. The collected data were analyzed by SPSS and related statistical methods. The most important finding in this study was the positive significant relation between cultural intelligence of managers and their effectiveness.and also results showed that there was a positive significant relation among the factors of Meta-cognition, motivation and behavior of cultural intelligence and effectiveness of managers, but there was no significant relation between cognition factor and effectiveness.

Keywords: Intelligence, Cultural Intelligence, Effectiveness, Managers

\section{Introduction}

In $21^{\text {st }}$ century, business has found global dimensions and requires adopting effectively with people from different cultures. Cooperation has become a necessity in business. We usually need to deal with international affairs and people from other countries and cultures. Unlike fast modernization of the world, changes in culture are under gone slowly. For predictable future, cultural differences will remain as a key factor in interpersonal interactions and we have known it for a 
long time that effective interaction with others is the most important part of management. In a $\mathrm{n}$ increasingly competitive world, the managers who do not keep their skills updated, must accept serious possibility of dismissal. We also know that cultural intelligence is the key competence of management in 21st century (Thomas \& Inkson, 2008).

Cultural intelligence (CQ) represents advancement in the area of international human resources management and cross-cultural training.(MacNab, et all, 2011)

In other definitions, the Cultural intelligence includes perception of principles of inter-cultural interactions, development of a thoughtful approach in inter-cultural interactions and finally, providing adaptive skills and detecting of a treasure from appropriate behaviors, so that the individual becomes effective in inter-cultural situations (Thomas and Inkson, 2008).

According to Plum, cultural intelligence includes three factors of inter-cultural requirement, inter-cultural communications and cultural perception. Generally, none of these dimensions work by itself, because they are dependant. Cultural intelligence also includes a continuous development of inter-cultural requirement and cultural perception as a position of preparation which leads to appropriate performance and creates a structure of cultural communication in current situation (Plum, 2008).

Cultural intelligence is the ability of showing effective reaction dealing with unknown cultures which contains different levels as well as social and emotional intelligence. Cultural intelligence enables people to perceive cultural differences via knowledge and accuracy; and takes proper behavior dealing with other cultures. The manager, with a high cultural intelligence, presents a behavior, by his experience, which exactly fights the current situation. (Thomas and Inkson, 2008).

Cultural intelligence (CQ), defined as a person's capacity in adapting new cultural contexts( Early and Ang, 2003,p 26), is an important driving expatriate adjustment and effectiveness. because individuals with a high level of CQ can more easily navigate and perceive unfamiliar cultures, theoretically, they are expected to be more successful when working and managing in countries except their native countries. (Ang et al, 2007)

Also, Ang et al (2007), suggested four dimensional cultural intelligence model and determined cultural intelligence including four factors of meta-cognition, cognition, motivation and behavior. (Ang et al, 2007)

A) Motivation: Motivational CQ is the ability to direct attention and energy toward learning about and working in culturally dissimilar situations(Rockstuhl et al 2011)

Motivational CQ refers to an individual's capability to direct attention and energy toward learning about and function in situations characterized by cultural differences. (Ang and Van Dyne, 2008)

Motivational cultural intelligence consist of intrinsic motivation, extrinsic motivation and self-efficacy. intrinsic motivation :the degree to which you derive enjoyment from culturally diverse situations, extrinsic motivation:the more tangible benefits you gain from culturally diverse experiences. self-efficacy: your confidence that you will be useful in a cross-cultural encounter.

All three of these motivational dynamics play a role in how leaders approach cross-cultural situations. Stop and examine your level of force to do cross-cultural work. Your motivational CQ is strongly related to your level of effectiveness in new cultural contexts. (Van Dyne et al 2009)

B) Cognition: Cognition or knowledge means the person's understanding of the issues and differences between cultures. Cognition is a knowledge dimension in Cultural intelligence and changes according to understanding level of leader about culture and the role of culture in shaping approach of an environment to next one. (Van Dyne 2009)

These knowledge structures provide them with a initial point to anticipate and understand cultural systems that shape and influence patterns of social interaction within a culture. (Rockstuhl et al, 2011)

C) Meta-Cognition: Cultural intelligence strategy or metacognitive cultural intelligence is the ability of using cultural knowledge to plan and to build an appropriate mental model for cross- cultural interactions; it includes awareness, planning and control .(Van Dyne et al, 2009)

"Awareness means being in tune with what's going on in one's self and others. Planning is taking the time to prepare for a cross-cultural encounter - anticipating how to approach the people, topic, and situation. Checking is the monitoring we do as we engage in interactions to see if the plans and expectations we had were appropriate". (Van Dyne et al, 2009)

D) Behavior: Behavioral cultural intelligence is practical aspect and deals with the staff and managers' capabilities in the appropriate performance in the scope of cross-cultural opportunities. (Ang et al, 2007)

The practical dimension of $\mathrm{CQ}$, refers to the leader's capability to act properly in a range of cross-cultural situations. It influences whether we can really achieve our performance goals effectively in light of different norms across cross-cultural situations. ( Van Dyne et al, 2009)

This aspect includes the flexibility in behaviors and verbal and non-verbal performance. Appropriate flexibility in 
eloquence is like applying of accurate words and sentences and non-verbal skills in communications in order to deliver special messages, with contents of this cultural intelligence (Ang et al, 2007).

Effectiveness:

Despite many studies on the effectiveness, uncertainty about what it is and what measures should be used to evaluate, is not yet fully resolved. Effectiveness means the "impact rate" used for individuals and actions. Effectiveness is also referred to as the ability to generate, product, penetration, decent, strong ... (Shirazi, 1994).

Peter Drucker interprets effectiveness as doing the right thing and efficiency as doing the thing rightly. But how can this right thing be measured, Daft suggests the following methods: (Seyedjavadin, 2004) (Table1)

Table 1. Methods of Effectiveness Measurement

\begin{tabular}{|l|l|l|}
\hline Methods & Topics & Description \\
\hline \multirow{4}{*}{ Traditional } & Goal-based & $\begin{array}{l}\text { The aim and purpose of the provision is determined. Index is operational objectives } \\
\text { and the limitations are multiple targets. }\end{array}$ \\
\cline { 2 - 3 } & Resource-based & $\begin{array}{l}\text { Effectiveness is based on supplying the resources from environment. This method is } \\
\text { used when the objectives are not recognizable and measurable. }\end{array}$ \\
\cline { 2 - 3 } Simultaneous & $\begin{array}{l}\text { The rate of integration and coordination of intra-organizational processes and } \\
\text { satisfaction of individuals- indexes of human relation such as cooperation, trust and } \\
\text { communication, beside economic efficiency, i.e. outcome to income, is presented. }\end{array}$ \\
\cline { 2 - 3 } & Organizational Process-based & $\begin{array}{l}\text { The role of organization in society on satisfying the stakeholders, including owners, } \\
\text { staff, customers, creditors, suppliers and government is focused as the main factor. }\end{array}$ \\
\cline { 2 - 3 } & Stakeholders' satisfaction & $\begin{array}{l}\text { The emphasis is on different aspects which are focused (internal or external) and } \\
\text { organizational structure (completely controlled flexibility). }\end{array}$ \\
\hline
\end{tabular}

As Sarcheshmeh copper mine is one of the largest copper mines in the world and the largest open pit mine in the Middle East, assessment the effectiveness of managers and cultural intelligence of them is mandatory and essential.

According to the four-factor model of cultural intelligence provided by Ang and Van Dean( Ang etal 2007), suggesting the components of cultural intelligence including cognition, metacognition, motivation and behavior, as well as the effectiveness of management components provided by Kreitner and Krinicki which know the effectiveness containing "performance, motivation, return on investment, delegation, leadership, and conflict management"( Kreitner and Krinicki 2009) and In regard to the importance of cultural intelligence, we investigated the relation of cultural intelligence of managers with their effectiveness in Sarcheshmeh Copper Complex in Kerman.

\section{Methodology}

The present study aimed to survey the relation between cultural intelligence of managers and their effectiveness in Sarcheshmeh Copper Complex in Kerman since Winter 2009 until Fall 2010. Sarcheshmeh Copper Complex of Kerman located in southern Iran. Population included 435 people of all managers (manager, boss, supervisor) of Sarcheshmeh Copper Complex. Krejes \& Morgan formula was used for sample volume calculation and 184 people were selected. The questionnaires were distributed withing the sample group through random categorical sampling method that is presented in table below.(Table 2)

Table 2. Sample Selection

\begin{tabular}{|c|c|c|c|}
\hline No. & Organizational Post & Amount & Sample Volume \\
\hline 1 & Manager & 38 & 16 \\
\hline 2 & Boss & 87 & 37 \\
\hline 3 & Supervisor & 310 & 131 \\
\hline \multicolumn{2}{|c|}{ Total } & 435 & 184 \\
\hline
\end{tabular}

In order to collect data two questionnaires were used: "cultural intelligence managers were assessed in four dimensions of metacognition, cognition, motivation and behavior, with 21 five-choice questions from completely disagree to strongly agree and measure of effectiveness of managementwith 22 five-choice questions in performance, leadership, authority, return on investment, conflict management, motivation and behavior from fully disagree to strongly agree". The questionnaire included two parts of demographic information and main questions. After the reliability and validity of the questionnaires were determined, they were distributed within the sample group. For validity, the questionnaires were 
given to five professors. After determining their ideas, and the validity was calculated in the frame of completely perfect, perfect, fair, poor and very poor. To determine the validity of the questionnaires, they were distributed among ten experts and after taking their view points, The validity of questionnaires was calculated.The validity of cultural intelligence questionnaire 0.88 and effectiveness questionnaire was 0.92 . To calculate reliability, the method of dual-test was used; i.e. the questionnaires were first distributed among ten people of the population and collected, and then again after ten days, it was repeated for the same 10 people. After comparing and calculating the reliability, it was $86 \%$ for cultural intelligence, and reliability of efficiency questionnaire was $89 \%$.

In this study, cultural intelligence of management was considered as independent variables and management effectiveness as the dependent variable; and the mediator variables include age, work experience, education level and gender. For data analysis, descriptive statistics (tables, graphs, frequency) and deductive analysis such as Spearman and Pearson tests and log-linear method were used. Data were analyzed using SPSS software.

\section{Results}

Table 1 shows the demographic findings of the patients.Based on obtained information, the results showed that $86.4 \%$ of respondents were male. This issue shows low rate of women in managerial level of organization which is common in all organizations of Iran.(Table 3)

Table 3. Socio-demographic data of the sample

\begin{tabular}{|l|c|cc|}
\hline Variables & & $\mathrm{N}$ & $\%$ \\
\hline Gender & Male & 159 & 86.4 \\
& female & 16 & 8.7 \\
\hline Age & $\leq 30$ & 19 & 10.3 \\
& $31-40$ & 86 & 46.7 \\
& $41 \geq$ & 72 & 39.1 \\
\hline Education Level & Diploma and less & 2 & 1.1 \\
& Assistents & 25 & 13.6 \\
& Bachelor & 128 & 69.6 \\
& Masters and higher & 22 & 12 \\
\hline Job Experiences & $<5$ Years & 10 & 5.4 \\
& 5-10 Years & 35 & 19 \\
& $11-20$ Years & 96 & 52.2 \\
& $21 \geq$ & 37 & 20 \\
\hline
\end{tabular}

Correlation analysis was used to examine the relationship between cultural intelligence and effectiveness of managers. At the error level of 0.05 and as the $p$ value was less than $5 \%$ and close to zero and Pearson and Spearman correlation coefficients were $329 / 0=r$ and $303 / 0=r$, the results showed that there was a significant positive relationship between cultural intelligence and effectiveness of the managers in population (Table 4).

Table 4. Results of Spearman and Pearson Correlation Tests for correlation of cultural intelligence and effectiveness

\begin{tabular}{|l|c|c|}
\hline & & Effectiveness of managers \\
\hline \multirow{2}{*}{ Cultural intelligence } & Pearson Correlation Coefficient & 0.329 \\
\cline { 2 - 3 } & P Value & 0.000 \\
\hline \multirow{2}{*}{ Cultural intelligence } & Spearman Correlation Coefficient & 0.303 \\
\cline { 2 - 3 } & P Value & 0.000 \\
\hline Number & \multicolumn{2}{|c|}{156} \\
\hline
\end{tabular}

Also, table 4 showed that, at the error level of 0.05 , and since the value of $p$ is less than $5 \%$ and close to zero, with $r=0.355$ and $r_{s}=0.360$ for metacognition and $r=0.272$ and $r_{s}=0.253$ for motivation and $r=0.275$ and $r_{s}=0.247$ for behavior, it can be argued that there is a positive relationship among the components of metacognition, motivation and behavior of cultural intelligence and effectiveness of managers in the studied population .

Furthermore, findings showed that correlation coefficient of $r=0.120$ and $r s=0.093$ and at the error level of 0.05 , and since the value of $p$ was greater than $5 \%(0.129)$, there was no significant relationship between the component of 
cognition and the effectiveness of managers in studied population. (Table 5)

Table 5. Results of Spearman and Pearson Tests for Correlation of Metacognition, Motivation, Behavior, cognition and Effectiveness of Managers

\begin{tabular}{|c|c|c|}
\hline & & Effectiveness of Managers \\
\hline \multirow[t]{4}{*}{ Meta cognition } & Pearson Correlation Coefficient & $0.355^{* \star}$ \\
\hline & P Value & 0.000 \\
\hline & Spearman Correlation Coefficient & 0.360 \\
\hline & $\mathrm{P}$ Value & 0.000 \\
\hline \multicolumn{2}{|r|}{ Number } & 158 \\
\hline \multirow[t]{4}{*}{ Motivation } & Pearson Correlation Coefficient & $0.272^{\star *}$ \\
\hline & P Value & 0.000 \\
\hline & Spearman Correlation Coefficient & 0.253 \\
\hline & P Value & 0.001 \\
\hline \multicolumn{2}{|r|}{ Number } & 161 \\
\hline \multirow[t]{4}{*}{ Behavior } & Pearson Correlation Coefficient & $0.275^{\star \star}$ \\
\hline & P Value & 0.000 \\
\hline & Spearman Correlation Coefficient & 0.247 \\
\hline & $\mathrm{P}$ Value & 0.001 \\
\hline \multicolumn{2}{|r|}{ Number } & 163 \\
\hline \multirow[t]{5}{*}{ Cognition } & Pearson Correlation Coefficient & 0.120 \\
\hline & P Value & 0.129 \\
\hline & Spearman Correlation Coefficient & 0.093 \\
\hline & P Value & 0.239 \\
\hline & Number & 162 \\
\hline
\end{tabular}

According to obtained median for the effectiveness of managers (4.1429), it can be concluded that managers in the studied population have a high effectiveness. Also obtained median for Cultural Intelligence of managers was 3.25, which represents the relatively high cultural intelligence of managers in studied population. Calculated averages also show that the components of metacognition, motivation and behavior of cultural intelligence of managers are relatively high, but the cognitive component of cultural intelligence is medium.

In order to investigate the relationship between cultural intelligence level of managers and their effectiveness with demographic variables, log-linear analysis was used. The results showed that gender, work experience, age and education level do not affect on the relationship between two variables of cultural intelligence of managers in Sarcheshmeh Copper Complexe and their effectiveness, and are independent of them.

\section{Discussion}

The main objective of this study was to identify and to describe the relationship between managers' cultural intelligence and their effectiveness in Sarcheshmeh Copper Complex. Results showed that there is a significant positive relationship between managers' cultural intelligence and their effectiveness in Sarcheshmeh Copper Complex.

Cultural intelligence experts' point of view including Thomas, Krinks, Early and Ang corresponded with the results, too.

On the other side, there is relationship between managers' effectiveness with cognitive, motivational, and behavioral intelligence as components of cultural intelligence.

Other studies have also been conducted in this area. Rockstuhl et al showed in their study that cultural intelligence was a stronger predictor of cross-border leadership effectiveness (Rockstuhl et al 2011)

Hadizadeh Moghaddam and Hosseini in their study concluded that there is a significant positive relationship between cognition, motivation, and behavior with team effectiveness (Hadizadeh Moghaddam and Hosseini 2008). Khani et al in their research on Mobarakeh Isfahan Steel Company have found that there is a significant positive relationship between dimensions of cultural intelligence (cognition, metacognition, motivation, and behavior) with team effectiveness. (Khani et al 2011)

Amiri et al concluded that there is a significant relationship between cultural intelligence components including metacognitive, cognitive, motivational, and behavioral as well as cultural intelligence itself with employees' performance. 
(Amiri et al 2010)

Rahimnia et al showed in their study that cognition and behavioral dimensions of cultural intelligence have positive relation to task performance.(Rahimnia et al 2010)

According to the results, there is a significant relationship between managers' metacognitive level as one components of cultural intelligence and their effectiveness in Sarcheshmeh Copper Complex.

As Triandis (2006) pointed out, persons with high metacognitive CQ have heightened perception of how their own culture influences their behavior and their understanding of intercultural situations. They also know the importance of preparing or planning for intercultural interactions by exposing themselves to different cultural norms. (Triandis 2006)

"Metacognitive CQ emphasizes strategy and is the lynchpin between understanding cultural issues and actually being able to use that understanding to be more effective". (Van Dyne 2009)

Motivational cultural intelligence also had a positive relationship with effectiveness. Motivational cultural intelligence reflects the ability and willingness to learn and work in different cultural situation (Ang et al, 2006).

Indivitual with high motivational CQ are attracted to intercultural situations because they value the benefits of these interactions and are sure that they can handle the intrinsic challenges of cultural differences(Van Dyne et al 2012)

Behavioral cultural intelligence also had significant positive relationship with managers' effectiveness.

Those with high behavioral CQ reveal flexibility in their intercultural interactions and adjust their behaviors to put others at ease and facilitate effective interactions.( Rockstuhl et al 2011)

Cognitive cultural intelligence had no significant relationship with managers' effectiveness in Sarcheshmeh Copper Complex. The results corresponded with the study conducted by Moshabaki and Tizro, also the results are inconsistent with researches conducted by Hadizadeh Moghaddam and Hosseini as well as Amiri et al.( Moshabaki and Tizro 2009, Hadizadeh Moghaddam and Hosseini 2008, Amiri et al 2010)

\section{Conclusion}

Based on results of this study as well as positive relationship between cultural intelligence and managers' effectiveness, looks particular investment needs to increase managers' effectiveness. Because increasing managers' cultural intelligence is possible through education and training, it is suggested that organizations devote special importance to improve cultural intelligence in their training and to develop programs for managers and to take steps to improve managers' cognitive and behavioral skills by using formal and informal training. Moreover, considering positive relationship between metacognitive intelligence and managers' effectiveness, more attention could be paid to training systems to improve analyzing skills so that metacognitive cultural intelligence will be improved.

\section{Acknowledgement}

Authors wish to thank the staff of Sarcheshmeh copper complex for participating in the study and Research Department of Azad University of Rafsanjan.

\section{References}

Amiri A, Moghimi S.M and Kazemi M ( 2010) Studying the Relationship between Cultural Intelligence and Employees' Performance. European journal of scientific Research, 42(3):418-427

Ang S, Van Dyne Land Koh Ch (2006 ) Personality correlates of the four-factor model of cultural intelligence. Group \& Organization Management ,31: 100-123

Ang S, Nielsen T. M and van dyne L (2007) Cultural Intelligence:345- 349

Ang S, Van Dyne L, KohCh Ng KYand et al ( 2007) Cultural intelligence: Its measurement and effects on cultural judgment and decision making, cultural adaptation and task performance. Manage. Organ. Rev; 3(3): 335-371.

Colakoglu S, Lepak D.P and Hong Y (2006) Measuring HRM effectiveness: Considering multiple stakeholders in a global context. Human Resource Management; 16:209 - 218

Earley P. C and Ang S. (2003).Cultural Intelligence: Individual Interactions A cross -culturals .California, Stanford University press.

Francis M . (2008). Effective Management, http//www. Changing Minds . org

Khani A, Etebarian A and Abzari M (2011) The relationship between cultural intelligence and group effectiveness in Mobarakeh steel company" African Journal of Business Management;5(17): 7507-7510

Kreitner R and Krinicki A(2009) Organizational Behavior. McGraw-Hill Higher Education

MacNab B ,Brislin R and Worthley R(2012)Experiential cultural intelligence development: context and individual attributes. The International Journal of Human Resource Management;23(7): 1320-1341

Moshabaki A and Tizro A (2009) The impact of emotional intelligence and cultural intelligence on the success of world-class 
leaders. Management study;2(3).

Plum E ( 2008 ) Cultural Intelligence : the art of leading cultural complexity .London, Middlesex university press: 28

Rahimnia F,Mortazavi s and Delaram T (2010) Examine the effects of cultural intelligence on the task performance of managers.Journal of management Tomorrow;8(33):67-88

Rockstuhl T, Seiler S and Ang Sand et al (2011) Beyond General Intelligence (IQ) and Emotional Intelligence (EQ): The Role of Cultural Intelligence (CQ) on Cross-Border Leadership Effectiveness in a GlobalizedWorld.Journal of Social Issues; 67(4): 825--840

Seyedjavadin S.A ( 2004) Comprehensive overview of management and organization theories. negah danesh publication: 26

Shirazi A (1994) education management. Mashhad, jahad daneshghahi publication; 288

Thomas D.C and Inkson k (2008) cultural intelligence. Second edition,San Francisco, Berret Koehler publishers.

Triandis H. C (2006) Cultural intelligence in organizations. Group and Organization Management,31 , 20-26. doi:10.1177/105960 1105275253

Van Dyne L, Ang S and Livermore D (2009) Cultural Intelligence : A Pathway for Leading in a Rapidly Globalizing World, CasebookCQ Chapter

Van Dyne L, Ang S and Yee Ng K et al(2012) Sub-Dimensions of the Four Factor Model of Cultural Intelligence: Expanding the Conceptualization and Measurement of Cultural Intelligence. Social and Personality Psychology Compass:6(4): 295-313 